\title{
The Impact of Training Residents on the Outcome of Robotic-Assisted Sacrocolpopexy
}

\author{
Mohamed A. Bedaiwy, ${ }^{1,2}$ Mohamed Abdelrahman,, ${ }^{1}$ Stephanie Deter, ${ }^{1}$ Tarek Farghaly, ${ }^{1}$ \\ Mahmoud M. Shalaby, ${ }^{2}$ Heidi Frasure, ${ }^{1}$ and Sangeeta Mahajan ${ }^{1}$ \\ ${ }^{1}$ Division of Female Pelvic Medicine and Reconstructive Surgery, Departments of Obstetrics and Gynecology and Urology, \\ University Hospitals Case Medical Center, MAC 5034, 11100 Euclid Avenue, Cleveland, OH 44106, USA \\ ${ }^{2}$ Assiut University, Assiut School of Medicine, Assiut 71111, Egypt
}

Correspondence should be addressed to Sangeeta Mahajan, sangeeta.mahajan@uhhospitals.org

Received 25 July 2012; Revised 31 August 2012; Accepted 23 September 2012

Academic Editor: Amanda Nickles Fader

Copyright (C) 2012 Mohamed A. Bedaiwy et al. This is an open access article distributed under the Creative Commons Attribution License, which permits unrestricted use, distribution, and reproduction in any medium, provided the original work is properly cited.

\begin{abstract}
Objective. To evaluate the surgical outcomes of robotic-assisted sacrocolpopexy (RASCP) before and after the incorporation of hands-on training for urology and gynecology residents. Study Design. Forty-one patients underwent RASCP between December 2008 and March 2010 with one surgeon. RASCP was performed in the context of surgical repair of complex pelvic organ prolapse and/or stress urinary incontinence. The first 20 cases (group I) were performed exclusively by the attending surgeon. In the last 21 cases (group II), the urology resident performed a 50\% or more of the RASCP while the gynecology resident performed the supracervical hysterectomy. The primary outcome measure was vaginal vault support at 24 weeks postoperatively based on pelvic organ prolapse quantification (POP-Q). Results. Mean \pm SD operative time for the entire surgery including RASCP was $282.3 \pm 51.3 \mathrm{~min}$ and median EBL was $83.1 \pm 50.4 \mathrm{~mL}$. Patient demographics and stage of disease did not differ between groups. Procedure time, PACU time, blood loss, and intraoperative complications were similar between groups. Follow-up POP-Q evaluations demonstrated significant correction of all points on vaginal examination for both groups $(P<0.001)$. Conclusions. Incorporation of resident training during RASCP allows teaching of robotic surgery techniques in an effective manner without prolonging operative time or affecting the overall surgical outcome.
\end{abstract}

\section{Introduction}

Pelvic organ prolapse is a very common problem that causes an estimated one in ten women to undergo surgery, and an additional $30 \%$ of these women will undergo additional surgery for repeat prolapse [1]. As the population of the United States continues to age, the number of women seeking treatment for pelvic organ prolapse will only continue to grow. The goal of surgical repair of all vaginal vault prolapse is to restore the anatomy and maintain sexual function and durability [2]. While the gold standard for vaginal vault prolapse is an abdominal sacrocolpopexy, large advances have been made in technology to allow minimally invasive approaches to become a viable alternative for surgeons [3]. Additionally, patients are also requesting a minimally invasive approach for their surgery because of the shorter hospital stay, decreased postoperative pain, and better cosmesis [4].
Initially, laparoscopy was offered to patients as a mode of performing a minimally invasive sacrocolpopexy. While patients have a decreased morbidity compared to traditional open approaches, there are notable difficulties experienced by the surgeon $[3,5]$. Decreased range of motion, twodimensional vision, and a steep learning curve are some of the many factors that have led to the increased operative time associated with laparoscopic surgery and have limited its widespread adoption by many surgeons. An increased skill level is also required to perform laparoscopic suturing, which is made difficult by the limited range of motion of the laparoscopic instruments [5].

More recently, the Da Vinci Surgical System (Intuitive Surgical Inc., Sunnyvale, CA) has provided the features needed to make the minimally invasive sacrocolpopexies successful [6]. The robot offers three-dimensional vision, increased magnification, tremor filtering, and seven degrees 
of freedom with its instruments that make a roboticassisted sacrocolpopexy less difficult than using a traditional laparoscope. The technical aspects of a RASCP reflect those of an abdominal sacrocolpopexy [7].

As the RASCP becomes more widely adopted into practice, the importance of training the next generation of practitioners becomes apparent without neglecting gaining experience in the traditional abdominal and vaginal hysterectomy concomitant with sacrocolpopexy [8]. Robotic surgery credentials are now required in certain places and in the near future it will be required more widely [9]. The training of residents and fellows on the technique of RASCP is important in both urology [10] and gynecology [11]. Balancing education and patient care is central in any surgery, and careful attention to primum non nocere is essential [12]. This study looks to evaluate the outcomes of RASCP before and after the incorporation of hands-on training for urology and gynecology residents.

\section{Materials and Methods}

Data were extracted from the medical records of all patients who underwent robotic-assisted sacrocolpopexy at the University Hospitals Case Medical Center (UHCMC) between April 2008 and March 2010. The approval of the UHCMC Institutional Review Board was obtained. The following data were extracted from each patient's medical record: age; stage of prolapse, concomitant procedure(s), intraoperative and postoperative complications, operative time, blood loss, conversion to laparotomy, length of hospital stay, resident hands- on contribution, and followup.

Forty-one patients underwent RASCP between December 2008 and March 2010 with one surgeon. RASCP was performed in the context of surgical repair of complex pelvic organ prolapse and, in some patients, stress urinary incontinence. The first 20 cases (group I) were performed exclusively by the attending surgeon. In the last 21 cases (group II), 2 urology residents at the PGY 5 level performed a $50 \%$ or more of the RASCP while 2 gynecology residents at the PGY 4 level performed the supracervical or total hysterectomy when indicated.

Prior robotic experience of all surgeons included exposure to didactic and instructional videos encompassing principals of robotic surgeries with video demonstration of a wide variety of gynecologic procedures. Subsequently, a dry laboratory hands-on training with the robotic system was completed. In addition, robotic surgical skills were also acquired in the animal laboratory using the porcine model.

Concomitantly, all surgeons assisted at the operating table in a wide variety of robotic procedures. Finally, all surgeons participation as console surgeon in the procedures was based on a stepwise progression through various aspects of the surgery by performing tasks with variable complexities under the supervision of the attending surgeons for the 4 residents or the supervision of another experienced attending in a minimum of 15 robotic procedures that were considered as a learning curve.

\section{Surgical Technique}

After induction of general anaesthesia, patients were positioned in dorsal lithotomy position with both arms tucked by the side and a bean bag was adjusted to keep the arms and the shoulders in place. Pneumoperitoneum is usually induced using a Verres needle. A $12 \mathrm{~mm}$ trocar was placed $2-5 \mathrm{~cm}$ supraumbilically. Two $8 \mathrm{~mm}$ robotic trocars were placed bilaterally, $10 \mathrm{~cm}$ lateral to and at the level of the umbilicus. An accessory $10 \mathrm{~mm}$ trocar was placed in the left lower quadrant. Monopolar scissors were inserted through the right robotic trocar and a Plasma kinetic (PK) dissecting forceps was inserted through the left robotic trocar.

The peritoneal surface over the sacral promontory was then incised at the base of the sigmoid mesentery and it was carefully dissected down the periosteum to avoid injuring the median sacral vessels. An endoanal sizer was inserted transvaginally to identify the vaginal cuff and the peritoneum overlying the vaginal apex was similarly incised. The bladder was then dissected anteriorly to expose the anterior vaginal wall and the space between the vagina and rectum was dissected in a similar fashion.

After completing the dissection, a Y-shaped polypropylene mesh (Restorelle, Mypathy Medical, Raynham, MA) was introduced through the $10 \mathrm{~mm}$ accessory port. The Monopolar scissors was then changed to a needle driver and the Y-shaped mesh were sutured to the anterior, posterior, and the apex of the vagina using permanent ( $2-0$ Goretex, $\mathrm{W}$. L. Gore and Associates, Inc., Flagstaff, AZ) sutures. The other end of the mesh was then sutured to the sacral promontory using the same type of permanent suture. Afer suturing both ends the mesh was then adjusted to avoid redundancy or excessive tension. CystoUrethoscopic examination after administration of intravenous indigo carmine at the end of the procedure to ensure ureteric patency and bladder integrity was performed in all patients.

\section{Followup}

All patients were asked to come for followup at 6 weeks postoperatively. Subsequent followup visits were individualized thereafter. Records were reviewed up to 24 weeks postoperatively.

\section{Statistical Analysis}

Patient demographic and clinical characteristics were described among all cases and compared between group 1 cases (without trainee involvement) and group 2 cases (with trainee involvement) by the use of either the chi-square or Fisher's exact test for frequency data or nonparametric Mann-Whitney test. Surgical outcomes were compared between groups in a similar fashion. Preoperative and postoperative POP-Q values were described and comparisons were made between groups by the use of the Mann-Whitney test and analysis of variance. 
TABle 1: Patient/clinical demographics overall and by group, $P$ value is comparison between groups.

\begin{tabular}{|c|c|c|c|c|}
\hline & Overall & Group $1(n=20)$ & Group $2(n=21)$ & $P$ value \\
\hline Age (years), median (IQR) & $61.5(15)$ & $61(12)$ & $63(16)$ & .744 \\
\hline Race & & & & .395 \\
\hline Caucasian & $29(71 \%)$ & $12(60 \%)$ & $17(81 \%)$ & \\
\hline African American & $4(10 \%)$ & $3(15 \%)$ & $1(5 \%)$ & \\
\hline Hispanic & $1(2 \%)$ & $1(5 \%)$ & 0 & \\
\hline Unknown & $7(17 \%)$ & $4(20 \%)$ & $3(14 \%)$ & \\
\hline BMI, mean $(\mathrm{SD})$ & $28.6(12.7)$ & $29.0(25.9)$ & $27.1(9.3)$ & .754 \\
\hline Stage & & & & .488 \\
\hline III & $39(95 \%)$ & $20(100 \%)$ & $19(91 \%)$ & \\
\hline IV & $2(5 \%)$ & 0 & $2(9 \%)$ & \\
\hline Prior prolapse surgery (yes) & $2(5 \%)$ & 0 & $2(10 \%)$ & .488 \\
\hline Prior incontinence surgery (yes) & $6(15 \%)$ & $2(10 \%)$ & $4(19 \%)$ & .663 \\
\hline Burch & 1 & 1 & 0 & \\
\hline TVT & 2 & 0 & 2 & \\
\hline Rectus FS & 1 & 1 & 0 & \\
\hline Other & 2 & 0 & 2 & \\
\hline Menopause (yes) & $34(83 \%)$ & $18(90 \%)$ & $16(76 \%)$ & .410 \\
\hline \multicolumn{5}{|l|}{ Selected comorbidities } \\
\hline Diabetes & $3(7 \%)$ & $2(10 \%)$ & $1(5 \%)$ & \\
\hline COPD/Asthma & $2(5 \%)$ & $2(10 \%)$ & 0 & \\
\hline $\mathrm{CAD}$ & $1(2 \%)$ & $1(5 \%)$ & 0 & \\
\hline HTN & $10(24 \%)$ & $8(40 \%)$ & $2(10 \%)$ & \\
\hline Cancer & $4(10 \%)$ & $4(20 \%)$ & 0 & \\
\hline Current steroid use & $1(2 \%)$ & $1(5 \%)$ & 0 & .488 \\
\hline
\end{tabular}

\section{Results}

6.1. Patient Characteristics. Forty-one patients with stage III/IV prolapse underwent RASCP between December 2008 and March 2010. The first 20 patients were performed exclusively by the attending surgeon (Group I) and the following 21 patients' surgeries were performed by urology or gynecology residents (group 2). Overall, the mean age was 61.5 (15) years and mean BMI was 28.6 (12.7) kg/m². Both groups were comparable regarding their age, ethnicity, and BMI. Stage and history of prior prolapse and incontinence surgery were similar between groups. Eighty-three percent of patients' surgeries were menopausal. Selected comorbidities were present in 12 patients ( 9 in group 1 and 3 in group $2 ; P=0.033)$. Patients' characteristics were summarized in (Table 1).

6.2. Intraoperative Outcomes. Concomitant procedures were performed in $36(88 \%)$ patients. When comparing operative outcome measures, there was no significant difference in OR time, procedure time, estimated blood loss, and PACU time between the two groups (Table 2). In addition, bladder perforation was encountered in $1(2 \%)$ of patients of group 1. It was recognized and adequately repaired intraoperatively without adverse sequelae. Vaginal wall was accidentally opened in one patient of group 2 due to extremely thin vagina and was sutured with adequate reapproximation.
6.3. Postoperative Outcomes. Postoperative complications are described in Table 2 . One patient in group 2 developed postoperative cuff dehiscence and was diagnosed 6 weeks postoperatively during routine postoperative follow-up visit. The vaginal cuff was revisited and adequately sutured under general anesthesia. One patient in group 1 required blood transfusion due to anemia secondary to chronic hemorrhoids in the postoperative period. Two patients in group 1 and one patient in group 2 were readmitted to the hospital for surgical repair of a vaginal mesh extrusion. Mesh extrusion is defined as any vaginal mesh exposure during the follow up period. All erosions were managed by freshening the edges and closing the vaginal defect. One patient required excision of a portion of the exposed mesh. Vaginal estrogen cream was offered to all patients after surgery. Three patients in group 1 developed postoperative urinary tract infection and were properly treated with antibiotics. Prolapse recurrence was reported in one patient of group 1 where the anterior vaginal wall was prolapsed to the level of the hymen. This patient underwent vaginal McCall culdoplasty. One patient in group 2 was complicated by postoperative ileus diagnosed with a CT scan. The patient was managed conservatively and showed a significant improvement on day 6 where she was discharged. One patient in group 2 developed postoperative surgical emphysema and pulmonary edema and she was readmitted to surgical intensive care unit (SICU) where she was properly 
TABLE 2: Surgical outcomes overall and by group.

\begin{tabular}{|c|c|c|c|c|}
\hline & Overall $(n=41)$ & Group $1(n=20)$ & Group $2(n=21)$ & $P$ value \\
\hline Concomitant procedures & $36(88 \%)$ & $18(90 \%)$ & $18(86 \%)$ & .999 \\
\hline TVT & $27(66 \%)$ & $12(60 \%)$ & $15(71 \%)$ & \\
\hline $\mathrm{SCH}$ & $22(54 \%)$ & $12(60 \%)$ & $10(48 \%)$ & \\
\hline Posterior repair & $9(22 \%)$ & $2(10 \%)$ & $7(33 \%)$ & \\
\hline Perineorraphy & $8(19 \%)$ & 0 & $8(38 \%)$ & \\
\hline TAH & $5(12 \%)$ & $3(15 \%)$ & $2(10 \%)$ & \\
\hline Enterocele & $1(2 \%)$ & $1(5 \%)$ & 0 & \\
\hline Other & $1(2 \%)$ & $1(5 \%)$ & $1(5 \%)$ & \\
\hline OR time (min), median (IQR) & $328.5(56)$ & $320(38)$ & $336(85)$ & .283 \\
\hline Range & $241-506$ & & & \\
\hline Procedure time (min), median (IQR) & $277(65)$ & $257(53)$ & $283(86)$ & .708 \\
\hline Range & $182-426$ & & & \\
\hline PACU time (min), median (IQR) & $97.5(61)$ & $90(80)$ & $110(45)$ & .444 \\
\hline Range & $52-335$ & & & \\
\hline Uterine weight $(\mathrm{g})$, mean $(\mathrm{SD}), n=19$ & $72.7(66.9)$ & & & \\
\hline EBL (cc), median (IQR) & $50(50)$ & $75(50)$ & $50(75)$ & .922 \\
\hline Range & $5-200$ & $25-175$ & $5-200$ & \\
\hline \multicolumn{5}{|l|}{$\mathrm{Hgb}, n=22$} \\
\hline Pre-operative & 13.2 & & & \\
\hline Post-operative & 11.4 & & & \\
\hline \multicolumn{5}{|l|}{$\mathrm{HCT}, n=22$} \\
\hline Pre-operative & 40.8 & & & \\
\hline Post-operative & 34.7 & & & \\
\hline Intraoperative complications & & & & .948 \\
\hline Perforation (bladder) & 1 & 1 & 0 & \\
\hline Vaginal wall defect & 1 & 0 & 1 & \\
\hline \multicolumn{5}{|l|}{ Post-operative complications } \\
\hline Urinary retention & 15 & 7 & 8 & \\
\hline Fever & 2 & 0 & 2 & \\
\hline Readmission to hospital & 3 & 2 & 1 & \\
\hline UTI & 3 & 3 & 0 & \\
\hline Transfusion & 1 & 1 & 0 & \\
\hline Cuff dehiscence & 1 & 0 & 1 & \\
\hline Failed voiding & 1 & 1 & 0 & \\
\hline Ileus & 1 & 0 & 1 & \\
\hline Prolapse recurrence & 1 & 1 & 0 & \\
\hline Emphysema, pulmonary edema & 1 & 0 & 1 & \\
\hline
\end{tabular}

managed and was discharged after 2 days. The mean length of hospital stay was 1.8 days (range 1-6 days) in both groups.

Preoperative POP-Q scores were similar between groups for anterior, apex, gh, pb, and TVL values (Table 3 ). There was a borderline significant difference $(P=0.057)$ between posterior (Ap and $\mathrm{Bp}$ ) scores between groups. On 12-week followup, the POP-Q values were significantly improved after surgery in both groups (Table 3, time effect) with no effect on vaginal length in both groups $(P=0.99)$. There was no interaction effect between group and time in POP-Q measurements; however, there was limited ability to detect differences due to small sample sizes.

\section{Discussion}

This study demonstrates that the incorporation of resident training does not appear to affect the immediate operative outcome on performing complex pelvic reconstructive surgery. This is important because the use of robotic-assisted sacrocolpopexy has given patients an alternative treatment to vaginal vault prolapsed [7]. In addition, RASCP is often the only option for patients whose age and medical comorbidities may make them less than ideal candidates for open surgery [7]. Initial studies have shown that initial durability of RASCP is similar to that of abdominal sacrocolpopexies 
TABLE 3: Mean preoperative and postoperative POP-Q values by group.

\begin{tabular}{cccccc}
\hline \multicolumn{5}{c}{ Preoperative } & \multicolumn{2}{c}{ Postoperative } & P value \\
& Group 1 & Group 2 & Group 1 & Group 2 & (time effect) \\
\hline Anterior & & & & & \\
Aa & +2.3 & +1.6 & -2.7 & -2.9 & $<.001$ \\
Ba & +3.6 & +2.7 & -2.7 & -2.9 & $<.001$ \\
Apex & & & & & \\
C & -2.6 & -2.1 & -9.7 & -9.8 & $<.001$ \\
Posterior & & & & & \\
Ap & -1.7 & -0.5 & -2.5 & -2.7 & $<.001$ \\
Bp & -1.7 & -0.2 & -2.5 & -2.8 & $<.001$ \\
gh & +3.8 & +3.7 & +3.2 & +3.1 & $<.001$ \\
pb & +2.4 & +2.2 & +3.3 & +3.3 & $<.001$ \\
TVL & +9.6 & +9.6 & +9.6 & +9.8 & 0.99 \\
\hline
\end{tabular}

${ }^{7}$ Pre-operative POP-Q scores were similar between groups. However, the closest to a borderline significant difference was in the posterior wall (Ap and Bp) scores $(P=.057)$.

[6]. There is only one study that reported a good patient satisfaction after one year followup after RASCP [13]. More studies are still needed to look at the long-term success of RASCP.

RASCP is still in its earlier stages of development. There are some negative consequences of RASCP that have emerged including increased mesh extrusion and cuff dehiscence. This is thought to be due to the amount of cautery used at the vaginal cuff particularly if a hysterectomy is done at the time of mesh placement during the RASCP [14]. Approximately $4 \%$ of patients will experience dehiscence of the vaginal cuff with the median presentation time of 43 days [2]. Our findings showed only one patient in forty-one (2\%) with cuff dehiscence. Advances in the types of mesh and suture used may affect outcomes in the future.

The limitation of this study is its retrospective design. All data was collected through medical records. This left a potential for misclassification bias, but we would not expect it to be different between the two groups.

One of the strengths of our study is the use of objective data to determine postoperative outcomes. POP-Q scores determined by the attending physician on 2 occasions (the initial encounter and during the preoperative visit) minimized the bias and discrepancy that could be prevented in the retrospective data.

As more physicians become trained in RASCP, the technique has been introduced to residents and fellows. While there is agreement that the procedure requires some degree of advanced laparoscopic skills, those used for the robot are often simpler than those used in laparoscopy [3, $6,15]$. The learning curve by the pioneers of RASCP was approximately fifty robotics cases [15]. More recent studies have shown that operative time improves after as few as ten cases [16]. The median operative time reported in our study was 277 minutes. This is similar to other studies that report operative times ranging from 172 minutes to 242 minutes [7]. The increased operative time is not solely related to resident training. The studies with the shortest operative times did not have any concurrent surgeries being performed at the time of the RASCP. This differs largely from our data in which $88 \%$ of patients had a concomitant surgery. In agreement with our data, Benson and colleagues reported 284 minutes operative time for Supracervical Roboticassisted Laparoscopic Sacrocolpopexy versus 194 minutes Robotic-assisted Laparoscopic Sacrocolpopexy [17]. In the future, it might be possible to compare patients undergoing only RASCP to obtain a more accurate time of resident operative times.

Minimally invasive surgery will only become more common in the future [1]. Residency training programs must use all opportunities to train residents and fellows on robotic surgery [16]. The quicker learning curve of the robot allows residents and fellows the chance to adopt the techniques they learn while in training and apply them in their future practices. As pelvic organ prolapse surgery volume increases, RASCP provides residents and fellows with an excellent opportunity to train on the robot safely and feasibly in a manner that does not affect patient morbidity $[8,12]$. Longterm data and robotic training consoles will only help in the development of such clinical training.

\section{Conflict of Interests}

The authors of this paper have nothing to declare.

\section{Acknowledgments}

The paper was presented in the 37 th annual meeting of the Society of Gynecologic Surgeons, San Antonio, TX, USA, April 11-13, 2011.

\section{References}

[1] G. Novara, A. Galfano, S. Secco, V. Ficarra, and W. Artibani, "Prolapse surgery: an update," Current Opinion in Urology, vol. 17, no. 4, pp. 237-241, 2007.

[2] M. L. Moy and S. Y. Byun, "Role of robotic surgery in urogynecologic surgery," Current Opinion in Urology, vol. 20, no. 1, pp. 70-74, 2010.

[3] J. Tan-Kim, S. A. Menefee, K. M. Luber, C. W. Nager, and E. S. Lukacz, "Robotic-assisted and laparoscopic sacrocolpopexy: comparing operative times, costs and outcomes," Female Pelvic Medicine \& Reconstructive Surgery, vol. 17, pp. 44-49, 2011.

[4] T. C. Chai, "Are costs for robotic assisted sacrocolpopexy lower than those for open sacrocolpopexy?" Journal of Urology, vol. 187, no. 2, pp. 644-645, 2012.

[5] P. Gadonneix, A. Ercoli, G. Scambia, and R. Villet, "The use of laparoscopic sacrocolpopexy in the management of pelvic organ prolapse," Current Opinion in Obstetrics and Gynecology, vol. 17, no. 4, pp. 376-380, 2005.

[6] D. S. DiMarco, G. K. Chow, M. T. Gettman, and D. S. Elliott, "Robotic-assisted laparoscopic sacrocolpopexy for treatment of vaginal vault prolapse," Urology, vol. 63, no. 2, pp. 373-376, 2004.

[7] E. J. Geller, N. Y. Siddiqui, J. M. Wu, and A. G. Visco, "Short-term outcomes of robotic sacrocolpopexy compared 
with abdominal sacrocolpopexy," Obstetrics and Gynecology, vol. 112, no. 6, pp. 1201-1206, 2008.

[8] D. Burkett, J. Horwitz, V. Kennedy, D. Murphy, S. Graziano, and K. Kenton, "Assessing current trends in resident hysterectomy training," Female Pelvic Medicine and Reconstructive Surgery, vol. 17, no. 5, pp. 210-214, 2011.

[9] B. K. Erickson, J. L. Gleason, W. K. Huh, and H. E. Richter, "Survey of robotic surgery credentialing requirements for physicians completing OB/GYN residency," Journal of Minimally Invasive Gynecology, vol. 19, no. 5, pp. 589-592, 2012.

[10] M. Robinson, A. MacNeily, L. Goldenberg, and P. Black, "Status of robotic-assisted surgery among Canadian urology residents," Journal of the Canadian Urological Association, vol. 6, no. 3, pp. 160-167, 2012.

[11] J. M. Gobern, C. M. Novak, and E. G. Lockrow, "Survey of robotic surgery training in obstetrics and gynecology residency," Journal of Minimally Invasive Gynecology, vol. 18, no. 6, pp. 755-760, 2011.

[12] F. Huettner, D. Dynda, M. Ryan, J. Doubet, and D. L. Crawford, "Robotic-assisted minimally invasive surgery; A useful tool in resident training - the Peoria experience, 20022009," International Journal of Medical Robotics and Computer Assisted Surgery, vol. 6, no. 4, pp. 386-393, 2010.

[13] D. S. Elliott, A. E. Krambeck, and G. K. Chow, "Long-term results of robotic assisted laparoscopic sacrocolpopexy for the treatment of high grade vaginal vault prolapse," Journal of Urology, vol. 176, no. 2, pp. 655-659, 2006.

[14] J. P. Gilleran, M. Johnson, and A. Hundley, "Robotic-assisted laparoscopic mesh sacrocolpopexy," Therapeutic Advances in Urology, vol. 2, no. 5-6, pp. 195-208, 2010.

[15] J. P. Lenihan, C. Kovanda, and U. Seshadri-Kreaden, "What is the learning curve for robotic assisted gynecologic surgery?" Journal of Minimally Invasive Gynecology, vol. 15, no. 5, pp. 589-594, 2008.

[16] M. N. Akl, J. B. Long, D. L. Giles et al., "Robotic-assisted sacrocolpopexy: technique and learning curve," Surgical Endoscopy and Other Interventional Techniques, vol. 23, no. 10, pp. 23902394, 2009.

[17] A. D. Benson, B. A. Kramer, R. O. Wayment, and B. F. Schwartz, "Supracervical robotic-assisted laparoscopic sacrocolpopexy for pelvic organ prolapse," Journal of the Society of Laparoendoscopic Surgeons, vol. 14, no. 4, pp. 525-530, 2011. 


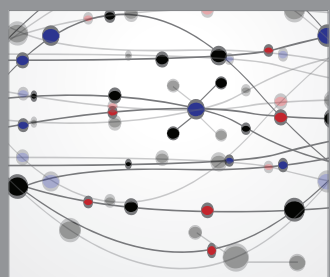

The Scientific World Journal
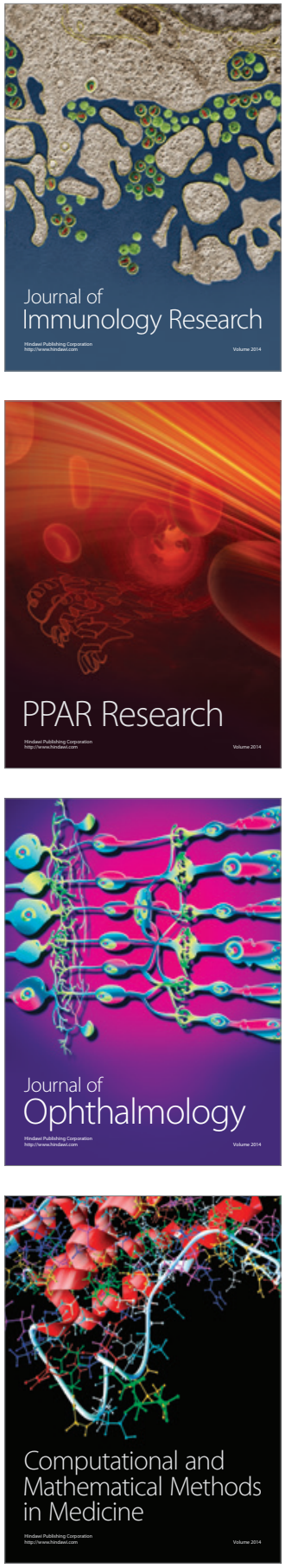

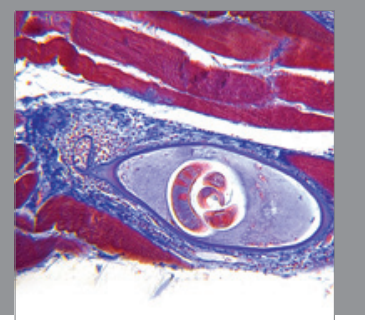

Gastroenterology

Research and Practice
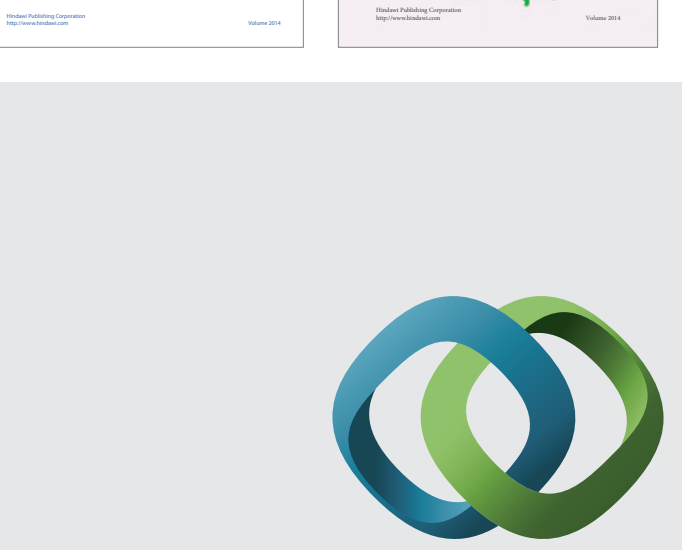

\section{Hindawi}

Submit your manuscripts at

http://www.hindawi.com
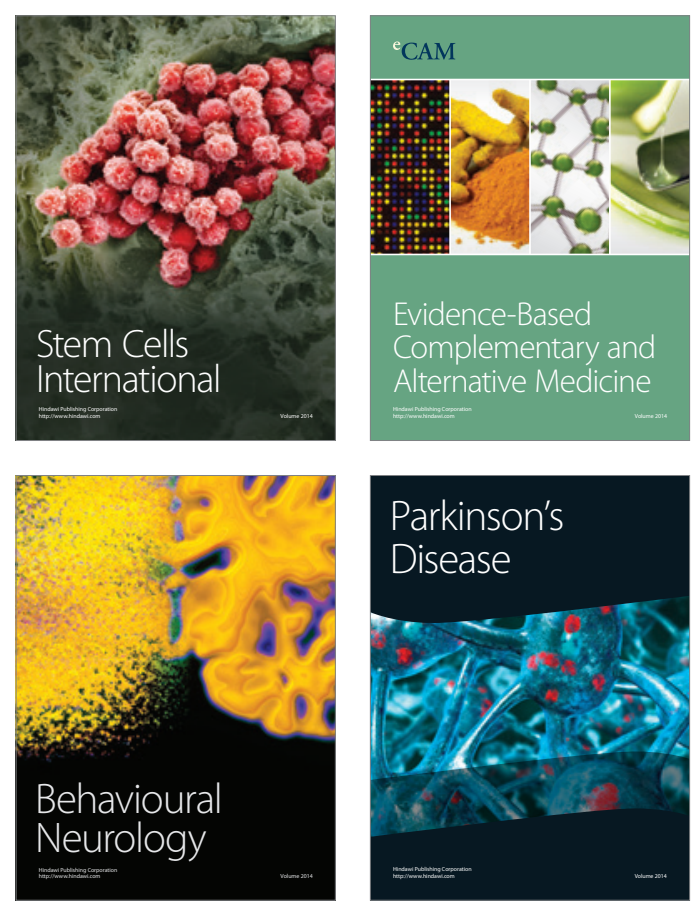

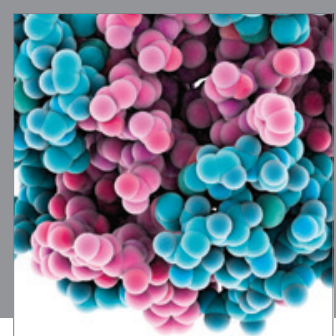

Journal of
Diabetes Research

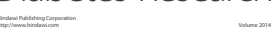

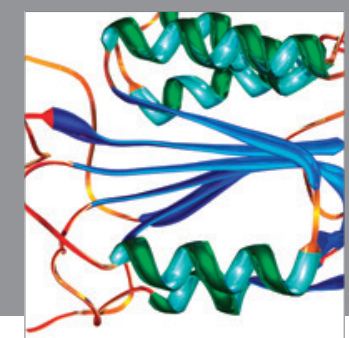

Disease Markers
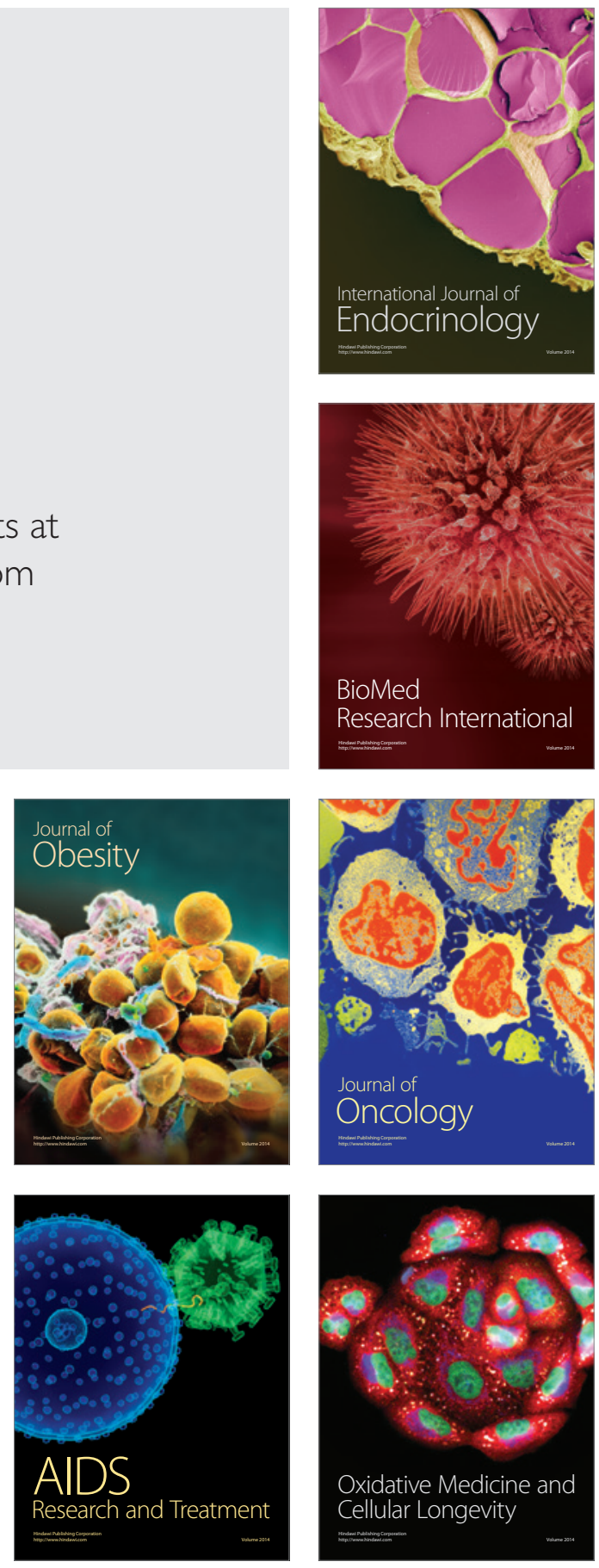